\title{
Cardiac troponin I-more than a biomarker for myocardial ischemia?
}

\author{
$\mathrm{Li} \mathrm{Ni}^{1,2,3,4}$, Xander H. T. Wehrens ${ }^{3,4,5,6,7}$ \\ ${ }^{1}$ Division of Cardiology, Department of Internal Medicine, Tongji Medical College Wuhan 430000, China; ${ }^{2}$ Hubei Key Laboratory of Genetics \\ and Molecular Mechanisms of Cardiological Disorders, Wuhan 430000, China; ${ }^{3}$ Cardiovascular Research Institute, ${ }^{4}$ Departments of Molecular \\ Physiology \& Biophysics, ${ }^{5}$ Departments of Medicine (Cardiology), ${ }^{6}$ Departments of Pediatrics (Cardiology), ${ }^{7}$ Center for Space Medicine, Baylor \\ College of Medicine, Houston, Texas, USA \\ Correspondence to: Xander H. T. Wehrens, MD, PhD, FAHA. Baylor College of Medicine, One Baylor Plaza, BCM335, Houston, TX, USA. \\ Email: wehrens@bcm.edu. \\ Comment on: Weil BR, Suzuki G, Young RF, et al. Troponin Release and Reversible Left Ventricular Dysfunction After Transient Pressure Overload. \\ J Am Coll Cardiol 2018;71:2906-16.
}

Submitted Aug 14, 2018. Accepted for publication Sep 07, 2018.

doi: 10.21037/atm.2018.09.07

View this article at: http://dx.doi.org/10.21037/atm.2018.09.07

Circulating cardiac troponin ( $\mathrm{c} T \mathrm{~T}$ ) concentrations have become an increasingly important biomarker for the noninvasive detection of myocardial injury in many cardiovascular diseases, in particular, patients with coronary heart disease presenting with acute chest pain (1). In this respect, elevated cTn levels are generally accepted to be a sign of myocyte necrosis, and considered as the goldstandard biomarker of myocardial infarction (2). However, elevated cTn is not only related to cardiomyocyte necrosis caused by coronary ischemia, but also found in a number of cardiac and non-cardiac conditions, such as heart failure (HF), pulmonary embolism, myopericarditis, kidney disease, sepsis, tachycardia, toxic injury, and even healthy people after high-intensity exercise (2-4).

As new and more sensitive cTn assays are being utilized, a substantial increase has been reported in a number of patients with detectable cTn increases, who were not diagnosed with acute coronary syndromes (2). Specifically, a growing body of evidence shows elevated levels of circulating $\mathrm{c} T \mathrm{n}$ in patients with $\mathrm{HF}$, in which the detection of cTn might be useful for risk stratification, HF prognosis, and therapies (5). However, there are still knowledge gaps in our current understanding of underlying mechanisms of elevated cTn released in acute and chronic HF. Multiple potential contributing mechanisms have been proposed, including cardiomyocyte necrosis, inflammation, oxidation, apoptosis and hibernating myocardium (5). Recently, studies demonstrate that cardiomyocytes can release cTnI by a stretch-related mechanism in the absence of necrosis (6).

In the author's previous study (7), they found elevated cTnI levels after transient ischemia without evidence of myocyte necrosis in a porcine model. These elevated cTnI levels were associated with delayed apoptosis, and no pathological evidence for myocardial infarction. Although it is unclear whether apoptosis and cTnI release are causally related to one another, these findings suggest that there maybe other mechanisms underlying the elevated serum levels of cTn in those non-ACS circumstances without cell necrosis.

In a recent article published in the fournal of the American College of Cardiology, Weil et al. (8) using a swine model investigated the pathophysiology of mechanical stretch-induced cardiomyocyte injury and the underlying mechanism of cTnI release. Left ventricular preload was raised by means of a transient arterial blood pressure increase by intravenous phenylephrine (PE) infusion $(300 \mathrm{mg} / \mathrm{min}$ ) for 1 hour. No ischemia occurred after $\mathrm{PE}$ assessed by microsphere flow measurements. Hemodynamics restored quickly after PE infusion, whereas $\mathrm{LV}$ ejection fraction remained reduced and normalized after 24 hours. Circulating cTnI elevated within 30 minutes and remained increased at 24 hours with pathological evidence showing myocyte apoptosis at 3 hours and normalized after 24 hours, consistent with myocardial stunning. These data demonstrate that elevated levels of circulating cTnI may be caused by LV preload-induced myocyte apoptosis in the 
absence of ischemia.

This translational study provides several new perspectives for us to better understand the mechanisms underlying clinical scenarios with c TnI elevations in the absence of ischemic heart disease. First, microsphere perfusion measurements showing normal subendocardial blood flow confirmed that temporary LV dysfunction was not induced by myocardial ischemia, but rather by myocardial stunning from mechanical stretch. Second, such transient LV dysfunction led to myocyte injury marked by reversible circulating cTnI elevations and myocyte apoptosis, without evidence of necrosis. These important results provide another explanation for $\mathrm{cTnI}$ elevations in patients without clinical evidence of cardiac ischemia. Troponin release can be triggered by ventricular volume overload, including heart failure, renal dysfunction, pulmonary embolism as well as others, in the absence of commonly assumed myocyte necrosis and ischemia. These findings may fill the gap in our understanding of the pathophysiology mechanisms of cTnI increase in non-ACS clinical conditions.

What is the translational relevance of these findings for patients with HF? While cTn levels are recommended biomarkers to identify the presence of an ACS, most patients with elevations of circulating cTn are not diagnosed with an ACS (9). Though it is difficult to identify specific mechanisms underlying various clinical disorders, it is believed that elevations of circulating c $\mathrm{Tn}$ reflect myocyte injury. In this regard, cTn is an "organ-specific" but not "disease-specific" biomarker (10). HF is caused by a complex set of interactions among various pathological processes leading to myocyte injury, mechanical stretch-induced injury, myocardial ischemia, and increased neurohormonal stimulation (5). Having a biomarker for several of these pathological processes including cardiomyocyte necrosis, apoptosis, myocyte injury from inflammation or oxidation, or apoptosis, may improve our ability to manage risk stratification and monitor progression of $\mathrm{HF}$ (5).

It is difficult to study cardiac changes during early stages of HF, in particular, during the transition from hypertrophy to cardiac dysfunction. The study by Weil et al. (8) suggests that $\mathrm{c} T \mathrm{n}$ plays a role in the transition process from hypertrophy to HF. However, in human HF, the elevations in intracardiac pressures persist for a long time, in contrast to the acute pressure elevation induced by a short period of PE injection in this study. Therefore, future studies are warranted to investigate the mechanism of different stages of $\mathrm{HF}$ in long-term animal models, which are applicable to both human mild and severe stages of HF. Furthermore, since the analysis of positive nuclei in TUNEL assays lack both sensitivity and specificity to identify apoptosis (11), more biochemical evidence of apoptosis is needed in future studies, such as the detection of Bcl-2 family proteins by immunoblotting, phosphatidylserine exposure by annexin $\mathrm{V}$ stain, and DNA fragmentation using Taq assay (11).

In conclusion, Weil et al. (8) showed that short-term elevated LV preload can induce delayed cTnl release and apoptosis without myocardial ischemia and necrosis. These findings are consistent with elevated cTnI levels observed in patients without clinical manifestation of cardiac ischemia. Elevated cTn levels can be used to detect and diagnose HF at an early stage, and are regarded as strong biomarkers for the prognosis of cardiovascular diseases. The findings published by Weil et al. (8) may help physicians diagnose $\mathrm{HF}$ and select adequate therapies to improve disease outcomes.

\section{Acknowledgements}

Funding: Dr. Ni is supported by National Natural Science Foundation of China (NSFC, No. 81470519), and Dr. Wehrens is supported by National Institutes of Health grants R01-HL089598, R01-HL091947, R01-HL117641, and R41-HL129570, a grant from the Saving Tiny Hearts Foundation, and the Quigley Endowed Chair.

\section{Footnote}

Conflicts of Interest: Dr. Wehrens is a founding partner of Elex Biotech, a start-up company that developed drug molecules that target ryanodine receptors for the treatment of cardiac arrhythmia disorders. Dr. Ni has no conflicts of interest to declare.

\section{References}

1. Thygesen K, Alpert JS, Jaffe AS, et al. Third universal definition of myocardial infarction. Circulation 2012;126:2020-35.

2. Giannitsis E, Katus HA. Cardiac troponin level elevations not related to acute coronary syndromes. Nat Rev Cardiol 2013;10:623-34.

3. Siriwardena M, Campbell V, Richards AM, et al. Cardiac biomarker responses to dobutamine stress echocardiography in healthy volunteers and patients with coronary artery disease. Clin Chem 2012;58:1492-4.

4. Predel HG. Marathon run: cardiovascular adaptation and 
cardiovascular risk. Eur Heart J 2014;35:3091-8.

5. Kociol RD, Pang PS, Gheorghiade M, et al. Troponin elevation in heart failure prevalence, mechanisms, and clinical implications. J Am Coll Cardiol 2010;56:1071-8.

6. Hessel MH, Atsma DE, van der Valk EJ, et al. Release of cardiac troponin I from viable cardiomyocytes is mediated by integrin stimulation. Pflugers Arch 2008;455:979-86.

7. Weil BR, Young RF, Shen X, et al. Brief Myocardial Ischemia Produces Cardiac Troponin I Release and Focal Myocyte Apoptosis in the Absence of Pathological Infarction in Swine. JACC Basic Transl Sci 2017;2:105-14.

8. Weil BR, Suzuki G, Young RF, et al. Troponin Release and Reversible Left Ventricular Dysfunction After Transient

Cite this article as: Ni L, Wehrens XH. Cardiac troponin Imore than a biomarker for myocardial ischemia? Ann Transl Med 2018;6(Suppl 1):S17. doi: 10.21037/atm.2018.09.07
Pressure Overload. J Am Coll Cardiol 2018;71:2906-16.

9. McFalls EO, Larsen G, Johnson GR, et al. Outcomes of hospitalized patients with non-acute coronary syndrome and elevated cardiac troponin level. Am J Med 2011;124:630-5.

10. Park KC, Gaze DC, Collinson PO, et al. Cardiac troponins: from myocardial infarction to chronic disease. Cardiovasc Res 2017;113:1708-18.

11. Takemura G, Kanoh M, Minatoguchi S, et al. Cardiomyocyte apoptosis in the failing heart--a critical review from definition and classification of cell death. Int J Cardiol 2013;167:2373-86. 